\title{
Exploring Anti-Fungal, Anti-Microbial and Anti- Inflammatory Properties of a Topical Non-Steroidal Barrier Cream in Face and Chest Seborrheic Dermatitis
}

\author{
Anna Balato (D) - Giuseppina Caiazzo - Roberta Di Caprio • \\ Emanuele Scala · Gabriella Fabbrocini · Corinne Granger
}

Received: September 20, 2019 / Published online: November 8, 2019

(C) The Author(s) 2019

\begin{abstract}
Introduction: The pathogenesis of seborrheic dermatitis (SD) is multifactorial and traditional treatments may not target all aspects of it. The aim of this study was to evaluate short-term anti-fungal, anti-microbial, anti-inflammatory and anti-pruritus properties of a novel nonsteroidal cream (NSC) containing piroctone olamine, zinc salt of L-pyrrolidone carboxylate (PCA), hydroxyphenyl propamidobenzoic acid, biosaccharide gum-2 and stearyl glycyrrhetinate in patients with face and chest SD.

Methods: Twelve male subjects affected by SD, presenting face and chest manifestations, were enrolled. Patients were instructed to apply NSC twice a day, performing regular visits at baseline (W0), after 7 (W1) and 14 (W2) days of treatment. A limitation of the study was that no
\end{abstract}

Enhanced Digital Features To view enhanced digital features for this article go to https://doi.org/10.6084/ m9.figshare.10033235.

A. Balato $(\varangle) \cdot$ G. Caiazzo

Department of Advanced Biomedical Sciences, University of Naples Federico II, Napoli, Italy

e-mail: annabalato@yahoo.it

R. Di Caprio · E. Scala · G. Fabbrocini

Department of Clinical Medicine and Surgery,

University of Naples Federico II, Napoli, Italy

C. Granger

Innovation and Development ISDIN, Barcelona, Spain control group treated with the vehicle without active ingredients was enrolled. To evaluate the efficacy of the NSC, investigator's assessments were represented by scoring index (SI) and investigator's global assessment score (IGA). In order to assess NSC anti-fungal and anti-microbial effects, skin scale scrapings were collected and used for Malassezia furfur (MF) and Staphylococcus epidermidis (SE) cultures. In parallel, in order to assess NSC anti-inflammatory effects, gene expression of IL- $1 \alpha$, IL-1 $\beta$, IL- 6 , IL- 8 , and TNF- $\alpha$ was assessed. In addition, anti-pruritus effects were also evaluated through gene expression of cathepsin $\mathrm{S}$ and L-histidine decarboxylase.

Results: SI mean scores significantly decreased at W1 and, to a greater extent, at W2 compared with W0. The IGA score registered an important improvement efficacy both for face and chest, from W1 to W2. MF and SE growth was already inhibited at W1, with a more pronounced decrease at W2. Gene expression of all analyzed mediators was significantly reduced at W1 compared to WO.

Conclusion: In conclusion, our assessment is that NSC is an effective and well tolerated treatment option for SD with anti-fungal, antimicrobial and anti-inflammatory properties.

Trial Registration: ISRCTN registry, ISRCTN77 871064 (retrospectively registered October 17, 2019). EudraCT number, 2019-003813-32.

Funding: ISDIN. 
Keywords: Biosaccharide gum-2; Hydroxyphenyl propamidobenzoic acid; Piroctone olamine; Malassezia; Seborrheic dermatitis; Staphylococcus epidermidis; Stearyl glycyrrhetinate; Zinc PCA

\section{Key Summary Points}

Why Carry Out This Study?

The main targets therapy of seborrheic dermatitis (SD) are the inflammation, the yeasts in particular Malassezia furfur, and recently also the microbial infection with Staphyloccocus epidermidis

Anti-fungal, anti-microbial and antiinflammatory properties evaluation of a novel non-steroidal cream (NSC) for SD treatment

What Was Learned from the Study?

This study has shown that NSC treatment was able to decrease Malassezia furfur and Staphyloccocus epidermidis growth as well as inflammatory cytokines

NSC is a valid treatment option for SD with anti-fungal, anti-microbial and antiinflammatory properties

\section{INTRODUCTION}

Seborrheic dermatitis (SD) is a common inflammatory skin disease affecting $1-3 \%$ of otherwise healthy individuals [1]. Men are affected more frequently than women $(3.0 \%$ vs $2.6 \%$ ) in all age groups, suggesting that SD may be associated with sex hormones such as androgens. Skin regions rich in sebaceous glands are commonly affected. SD often presents as pink to red greasy-looking skin with yellowish scales in seborrheic areas such as the nasolabial folds, upper lip, eyelids and eyebrows, scalp, retro-auricular areas and the upper chest [2-4]. Despite the high prevalence, the pathogenesis of SD is not well understood.
However, studies have identified several factors, including fungal colonization, sebaceous gland activity, as well as individual susceptibility. Numerous pieces of evidence suggest a pathogenic role for yeasts of Malassezia in SD [5, 6]. Malassezia are lipophilic yeasts found mainly on seborrheic regions of the body. Studies have detected Malassezia on the scalp of dandruff patients, and higher numbers of Malassezia correlate with SD appearance/severity [7, 8]. Furthermore, Malassezia were shown to have lipase activity, which hydrolyzes human sebum triglycerides and releases unsaturated fatty acids such as oleic and arachidonic acid $[9,10]$. These metabolites cause aberrant keratinocyte differentiation, resulting in stratum corneum abnormalities such as parakeratosis, intracellular lipid droplets, and irregular corneocyte envelopes [11]. Such changes lead to disrupted epidermal barrier function and trigger an inflammatory response, with or without visible local inflammation. In addition, these metabolites induce keratinocytes to produce pro-inflammatory cytokines such as interleukin (IL)-1 $\alpha$, IL-6, IL-8 and tumor necrosis factor (TNF)- $\alpha$, thus prolonging the inflammatory response $[1,12]$. Moreover, a recent study by Tanaka et al. showed a predominance of Staphylococcus on SD lesional sites. The authors suggest that, in addition to Malassezia, these commensal bacteria might contribute to SD development [13]. The therapeutic approach should be selected according to SD severity and the patient's immune status and compliance. For mild to moderate facial and chest SD, topical drugs play a key role in the management by reducing erythema and scale production. A variety of treatments including topical corticosteroids, antifungal agents, calcineurin inhibitors and, more recently, non-steroidal creams, are available [14]. To date, it is available a novel nonsteroidal cream (NSC) containing different ingredients, which have been reported to exert effects in SD treatment [14]. In particular, this cosmeceutical is composed of: (1) piroctone olamine, that has an anti-fungal property [15-17]; (2) zinc salt of L-pyrrolidone carboxylate (PCA), that has anti-fungal and anti-inflammatory properties [18-20]; hydroxyphenyl propamidobenzoic acid, that 
has anti-irritant and anti-itch properties [14]; (4) biosaccharide gum-2, that has anti-inflammatory and antifungal properties (patent pending 2019, WO2019020822) and soothing active properties [21]; and (5) stearyl glycyrrhetinate that has anti-inflammatory, antioxidant, and skin-soothing properties [22].The aim of this study was to evaluate shortterm anti-fungal, anti-microbial, anti-inflammatory and anti-pruritus properties of this NSC in patients with face and chest SD.

\section{METHODS}

\section{Study Population}

The overall study population included 12 male SD subjects presenting face and chest manifestations. SD patients were recruited from the Dermatology Unit of the University of Naples Federico II, Italy. The inclusion and exclusion criteria of SD patients are summarized in Table 1.

\section{Compliance with Ethics Guidelines}

The experimental protocol was approved the March14, 2018 by the Ethics Committee for Biomedical Activities "Carlo Romano" of University of Naples Federico II and conformed to the principles outlined in the Declaration of Helsinki of 1964, as revised in 2013, concerning human and animal rights. Each subject gave written informed consent before entering the study. The patient gave permission for his photograph to be published in the manuscript.

\section{Study Design}

This was an open-label, prospective and experimental research clinical trial. Patients were instructed to apply the non-steroidal cream commercialized as NUTRADEICA ${ }^{\circledR}$ (ISDIN Spain), containing piroctone olamine [\% International Nomenclature of Cosmetic Ingredients (INCI), 0.45-0.55], zinc PCA, hydroxyphenyl propamidobenzoic acid (\% INCI, 0.045-0.055), biosaccharide gum-2 (\% INCI, 0.045-0.055), and stearyl glycyrrhetinate (\% INCI, 0.27-0.33), twice daily on the face and on the chest, performing regular visits at baseline (W0), after 7

Table 1 Study inclusion and exclusion criteria

\begin{tabular}{|c|c|}
\hline Inclusion criteria & Exclusion criteria \\
\hline Diagnosis of SD on face and chest areas & Subjects participating in any other clinical study \\
\hline Males between 21 and 65 years of age & $\begin{array}{l}\text { Subjects having an acute or chronic disease or medical condition which could } \\
\text { put at risk or compromise study outcomes }\end{array}$ \\
\hline Fitzpatrick skin type I-IV & $\begin{array}{l}\text { History of allergic reactions, skin sensitization and/or known allergies to } \\
\text { cosmetic ingredients, toiletries, sunscreens, etc. }\end{array}$ \\
\hline $\begin{array}{l}\text { Subjects willing to sign informed } \\
\text { consent, to be photographed }\end{array}$ & Immunocompromised subjects (e.g., cancer, AIDS, etc.) \\
\hline \multirow[t]{2}{*}{ Subjects to accept skin biopsies } & $\begin{array}{l}\text { Patients taking other medications (oral or topical) which could interfere with } \\
\text { the interpretation of the results (not allowed: anti-inflammatory and anti- } \\
\text { fungal drugs, antibiotics, topical cosmetic compounds as well as oral } \\
\text { supplements) }\end{array}$ \\
\hline & $\begin{array}{l}\text { Washout from prior medications higher than } 3 \text { weeks for systemics and } 2 \text { weeks } \\
\text { for topical therapy }\end{array}$ \\
\hline
\end{tabular}


(W1) and 14 (W2) days of treatment. In addition, patients were instructed to not apply the cream the morning of the scheduled follow-up visits (W1, W2). Patients were evaluated through questionnaires filled out by both investigators and patients themselves. In particular, investigator's assessments were represented by scoring index (SI) and investigator's global assessment score (IGA). The SI ranking system, recommended by Koca et al. [23], was used at all visits (W0, W1 and W2). According to this system, erythema, scaling, itching and irritation of each area is ranked from 0 to 3 (non existence, 0 ; mild, 1 ; moderate, 2 ; severe, 3 ). The sum of these values is regarded as the SD rank: 0-4 (mild), 5-8 (moderate) and 9-12 (severe). IGA was performed at W1 and W2. According to this system, post-treatment rank was used to make a final evaluation of the recovery rate from 0 (no efficacy) to 10 (cured). In particular, the response was defined as excellent from 8 to 10, good from 5 to 7 , mild lower than 5 and no response or worsening as 0 . Regarding patients' assessments, they were represented by visual analog scales (VAS), patient global assessment (PGA), and subjective questionnaire for cosmetic and efficacy properties. VAS measurement was assessed at W0, W1 and W2. This score, ranging from 0 to $100 \mathrm{~mm}$, was used to assess each of the following symptoms (erythema, scaling, itching, hot sensation, pain and irritation) both for face and chest. PGA was assessed at W1 and W2. According to this system post-treatment rank was used to make a final subjective evaluation of the recovery rate from 0 (no efficacy) to 10 (cured). In addition, patients performed the subjective questionnaire for cosmetic and efficacy properties of the product at W1 and W2 (answers: completely disagree, disagree, agree, completely agree) composed by the following questions: (Q1, The product is well absorbed; Q2, The product has a pleasant texture; Q3, My skin tolerates the product well; Q4, The product acts quickly after being applied on my skin; Q5, The product applied is effective). Beyond characterizing patients from a clinical point of view in order to assess the efficacy of the product, patients underwent additional procedures to evaluate anti-fungal, anti-microbial and anti-inflammatory effects.

In order to assess NSC anti-fungal and antimicrobial effects, skin scale scrapings (50-100 $\mathrm{mg}$ ) from the face (wings of the nose) and from the chest were collected at W0, W1 and W2 to be used for Malassezia furfur (MF) and Staphylococcus epidermidis (SE) colony-forming units. In parallel, in order to assess NSC antiinflammatory effects, each recruited patient was biopsied ( $2 \mathrm{~mm}$ diameter) in the same area on the chest at W0 and W1 for gene expression analysis.

\section{Malassezia furfur Colony-Forming Units}

Skin scales (50-100 mg weight) were scraped from the face as well as the chest and cultured into Malassezia agar plates (Dixon's, $14.0193 \mathrm{~V}$ ) at $36^{\circ} \mathrm{C}$ in the dark for 7 days in order to assess the MF growth. Affected sites were cleaned with $70 \%$ ethyl or isopropyl alcohol before removing skin scales.

Cultured samples were examined every 2 days for growth. Indeed, $\mathrm{MF}$ at $36{ }^{\circ} \mathrm{C}$ after $48 \mathrm{~h}$ of incubation initially presented smooth, creamy colonies, from yellow to brownish, which over time took on a rough appearance. MF colonies were counted and reported as total colony-forming units per dish (cfu/dish).

\section{Staphylococcus epidermidis Colony- Forming Units}

Skin scales (50-100 mg weight) were scraped from face as well as chest and cultured on Chapman-Mannitol salt agar plates (Scharlau, 064-PR0015) at $37^{\circ} \mathrm{C}$ for $48 \mathrm{~h}$ in order to assess $\mathrm{SE}$ growth. This culture medium is specific for the isolation and enumeration of staphylococci. The selectivity of this medium is based on the presence of sodium chloride which inhibits the growth of most Gram (+) and Gram (-) bacteria. Differentiation of staphylococci is based on their capacity to ferment mannitol. Fermentation of mannitol induces acidification, which turns the medium yellow in the presence of phenol red ( $\mathrm{pH}$ indicator). Strains of SE form small colonies which, in the majority of cases, 
grow without modifying the color of the medium. SE colonies were counted and reported as total colony-forming units per dish (cfu/dish).

\section{Gene Expression Analysis}

RNA was extracted from skin biopsies performed at W0 and W1 (RNeasy Mini Protocol Qiagen, Valencia, CA) and cDNA was prepared (Transcriptor High Fidelity cDNA Synthesis, Roche, Indianapolis, IN) according to the manufacturer's instructions. qRT-PCR (LightCycler, Roche) was used to analyze the levels of expression of 18S (housekeeping gene) as well as all selected mediators (reported below). The PCR protocol and product quantification for all analyzed genes were performed as previously reported [24]. PCR primers for the selected genes were designed based on published sequences, and their specificity was verified with a BLAST alignment search. A melting curve analysis was carried out after completion to confirm the presence of a single amplified species. The amount of mRNA for a given gene in each sample was normalized to the amount of mRNA of $18 \mathrm{~S}$ reference gene in the same sample. Fold induction of gene expression was calculated using the $\Delta \Delta \mathrm{CT}$ method as described previously [25]. The analyzed genes were divided according to their function as follows, for anti-inflammatory effects: IL- $1 \alpha$, IL- $1 \beta$, IL- 6 , IL8 , and TNF- $\alpha$; for anti-microbial effects: HBD2 and HBD3, and for anti-pruritus effects: cathepsin S (CTS) and L-histidine decarboxylase (HDC).

\section{Statistical Analysis}

Statistical analyses were performed using GraphPad Prism 6.0 (GraphPad Software Inc., La Jolla, CA, USA). Data were analyzed with Wilcoxon matched-pairs tests to calculate statistical differences. Values of $p<0.05$ were considered significant. Data are expressed as the mean \pm SD.

\section{RESULTS}

\section{Clinical Effectiveness}

Enrolled patients were affected by moderate to severe $\mathrm{SD}$, since mean rank calculated according to Koca et al. [23], was 8.3. Face: SI mean scores of erythema, itch, scaling, and irritation significantly decreased at W1 and, to a greater extent, at W2 of the NSC treatment, compared with the baseline (W0) (Fig. 1a). In line with these results, mean VAS of all evaluated symptoms by patients (itch, erythema, scaling, irritation, pain, and hot sensation) also showed significant reduction at W1 and W2 (Fig. 1b). In particular, itch, erythema, scaling, and hot sensation showed a more pronounced decrease at W2 $(p<0.001)$. Chest: SI mean scores of erythema, itch, scaling, and irritation significantly decreased at W1 and, to a greater extent, at W2 of the NSC treatment, compared with WO (Fig. 1d). In line with these results, mean VAS of all evaluated symptoms by patients (itch, erythema, scaling, irritation, pain, and hot sensation) also showed significant reduction at W1 and W2 (Fig. 1f). In particular, itch, erythema, scaling, and irritation showed a more pronounced decrease at W2 $(p<0.001)$. The IGA score registered an important improvement efficacy both for face and chest, from W1 to W2 $(p<0.001)$ (Fig. 2a). In addition, the PGA score showed a significant improvement from $\mathrm{W} 1$ to W2 $(p<0.001)$ (Fig. 2b).

Clinical improvements were also evident in a clear manner from representative clinical images after 7 days (W1) of topical application of NSC on the face as well as on the trunk (Fig. 2c, d). Overall, no signs of local side effects were documented during the study and cosmetic tolerability and acceptability was rated as excellent by all patients, since most of the answers to the queries were 'agree' and 'completely agree,' as shown in Table 2.

\section{Antifungal and Antimicrobial Effects}

For the face, significant reduction of MF as well as SE cfu counts at W1 and W2 was observed (Fig. 3a, b). In particular, NSC treatment was 
Face

A

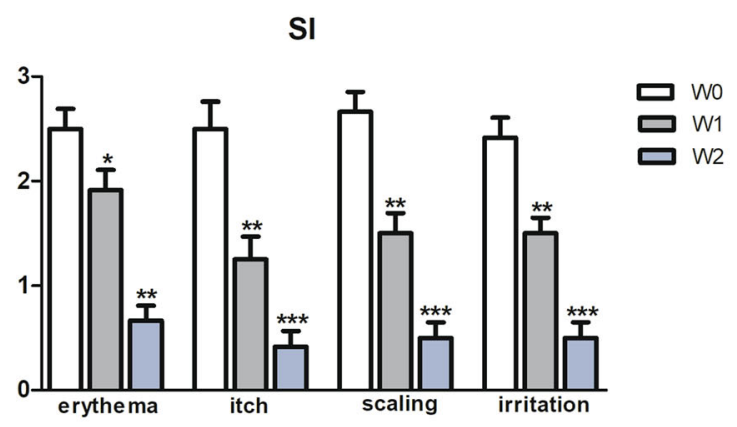

C

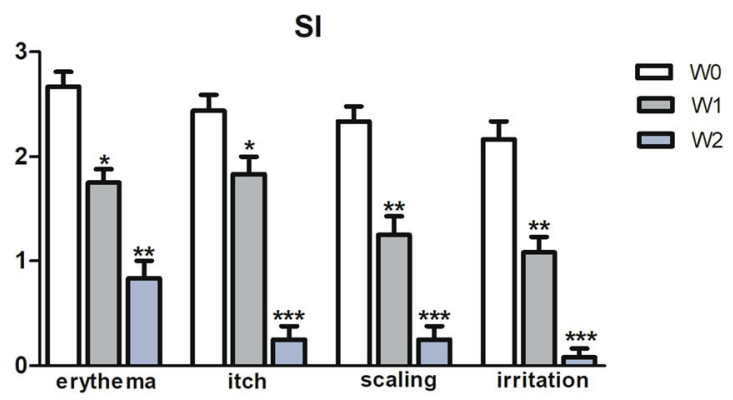

Fig. 1 Mean values of scoring index (SI) for erythema, itch, scaling and irritation on face and chest at baseline (W0), after 7 (W1) and 14 (W2) days of treatment (a, c). Mean values of visual analog scale (VAS, range $0-10)$ for

able to strongly decrease MF growth at W1 $(18 \pm 9 \mathrm{cfu}, \quad p<0.001)$ with respect to W0 $(250 \pm 12 \mathrm{cfu})$, registering a reduction of $92.8 \%$, with a greater inhibition at W2 $(5 \pm 3 \mathrm{cfu}$, $p<0.001$ ), registering a reduction of $98 \%$. A similar but less pronounced trend to decrease was also seen for SE growth at W1 $(1350 \pm 45 \mathrm{cfu})$ with respect to W0 $(1600 \pm 60 \mathrm{cfu})$, registering a reduction of $15.6 \%$, with a greater inhibition at W2 $(400 \pm 32 \mathrm{cfu}, p<0.01)$, registering a reduction of $75 \%$. For the chest, significant reduction of MF as well as SE cfu counts at W1 and W2 was observed. In particular, NSC treatment was able
B

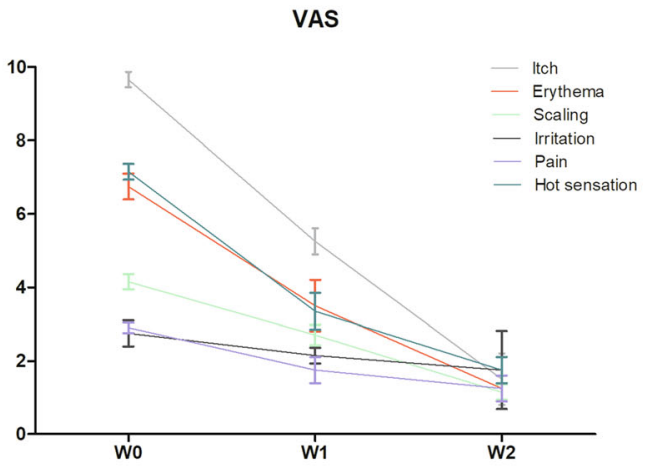

D

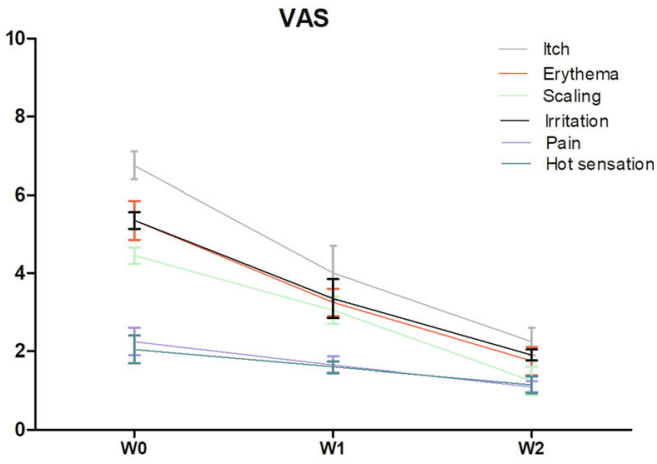

itch, erythema, scaling, irritation, pain and hot sensation at W0, W1 and W2 (b, d). Statistical analyses were performed by Wilcoxon matched pairs test $\left({ }^{*} p<0.05\right.$; $\left.{ }^{* *} p<0.01,{ }^{* * *} p<0.001\right)$

to strongly decrease MF growth at W1 $(60 \pm 13 \mathrm{cfu}, p<0.01)$ with respect to W0 $(380 \pm 23 \mathrm{cfu})$, registering a reduction of $84 \%$, with an almost complete inhibition at W2 $(4 \pm 1.5 \mathrm{cfu}, p<0.001)$, registering a reduction of $99 \%$. Similarly, SE growth was significantly reduced at $\mathrm{W} 1(65 \pm 7 \mathrm{cfu}, p<0.001)$ with respect to WO $(830 \pm 16 \mathrm{cfu})$, registering a reduction of $92 \%$, with an almost complete inhibition at W2 (6 $\pm 3 \mathrm{cfu}, p<0.001)$, registering a reduction of $99 \%$. Representative pictures of MF as well as SE cultures are displayed in Fig. 3. 
A

IGA

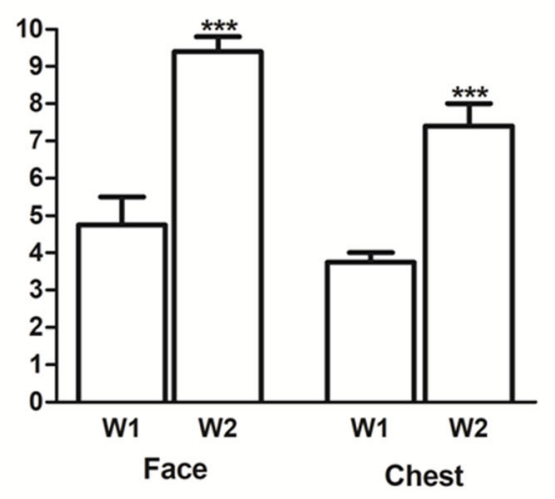

C
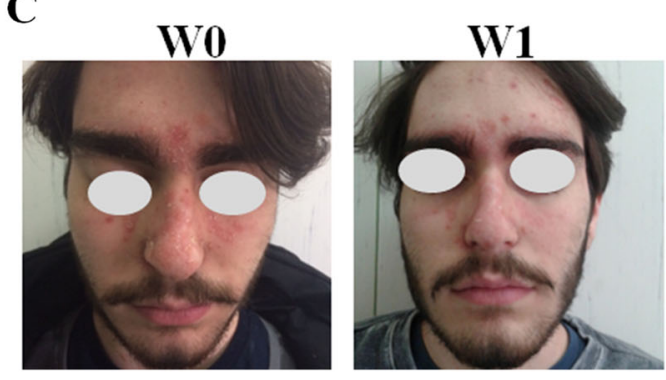

Fig. 2 Mean values of investigator's global assessment score of face and chest (IGA, range $0-10$ ) at W1 and W2 (a). Mean values of patient's global assessment score of face and chest (PGA, range $0-10$ ) at $\mathrm{W} 1$ and $\mathrm{W} 2$ (b).
B

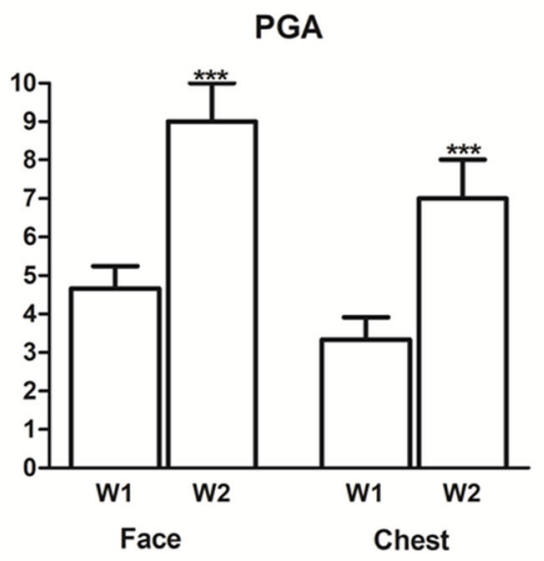

D

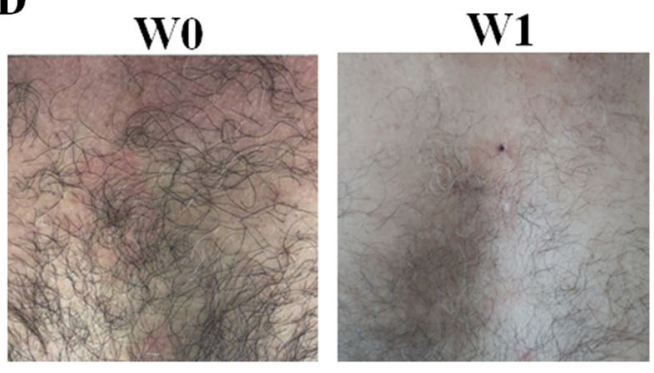

Statistical analyses were performed by Wilcoxon matched pairs test $\left.{ }^{* * *} p<0.001\right)$. Representative clinical images at W0 and W2 on face as well as chest $(\mathbf{c}, \mathbf{d})$

Table 2 Subjective questionnaire for cosmetic and efficacy properties of the product at W1 and W2

\begin{tabular}{|c|c|c|c|c|c|c|c|c|}
\hline \multirow[t]{2}{*}{ Query } & \multicolumn{2}{|c|}{$\begin{array}{l}\text { Completely } \\
\text { disagree (\%) }\end{array}$} & \multicolumn{2}{|c|}{$\begin{array}{l}\text { Disagree } \\
\text { (\%) }\end{array}$} & \multicolumn{2}{|c|}{$\begin{array}{l}\text { Agree } \\
(\%)\end{array}$} & \multicolumn{2}{|c|}{$\begin{array}{l}\text { Completely } \\
\text { agree (\%) }\end{array}$} \\
\hline & W1 & W2 & W1 & $\mathbf{W} 2$ & W1 & $\mathbf{W 2}$ & W1 & W2 \\
\hline The product is well absorbed? & 0 & 0 & 0 & 0 & 50 & 0 & 50 & 100 \\
\hline The product has a pleasant texture? & 0 & 0 & 0 & 0 & 62.5 & 0 & 37.5 & 100 \\
\hline My skin tolerates the product well? & 0 & 0 & 0 & 0 & 50 & 25 & 50 & 75 \\
\hline The product acts quickly after being applied on my skin? & 0 & 0 & 12.5 & 0 & 75 & 50 & 12.5 & 50 \\
\hline The product applied is effective? & 12.5 & 0 & 0 & 0 & 75 & 25 & 12.5 & 75 \\
\hline
\end{tabular}

\section{Gene Expression Effects}

Gene expression of all inflammatory mediators analyzed (IL-1 $\alpha$, IL-1 $\beta$, IL-6, IL-8, TNF- $\alpha$ ) was significantly reduced after 7 days of treatment (W1) compared to W0 (Fig. 4a). Gene expression of antimicrobial peptides (HBD2 and HBD3) was significantly decreased at W1 compared to W0 (Fig. 4b). Gene expression of pruritus mediators (CTS, HDC) was significantly decreased at W1 compared with W0 (Fig. 4c). 
Face Chest

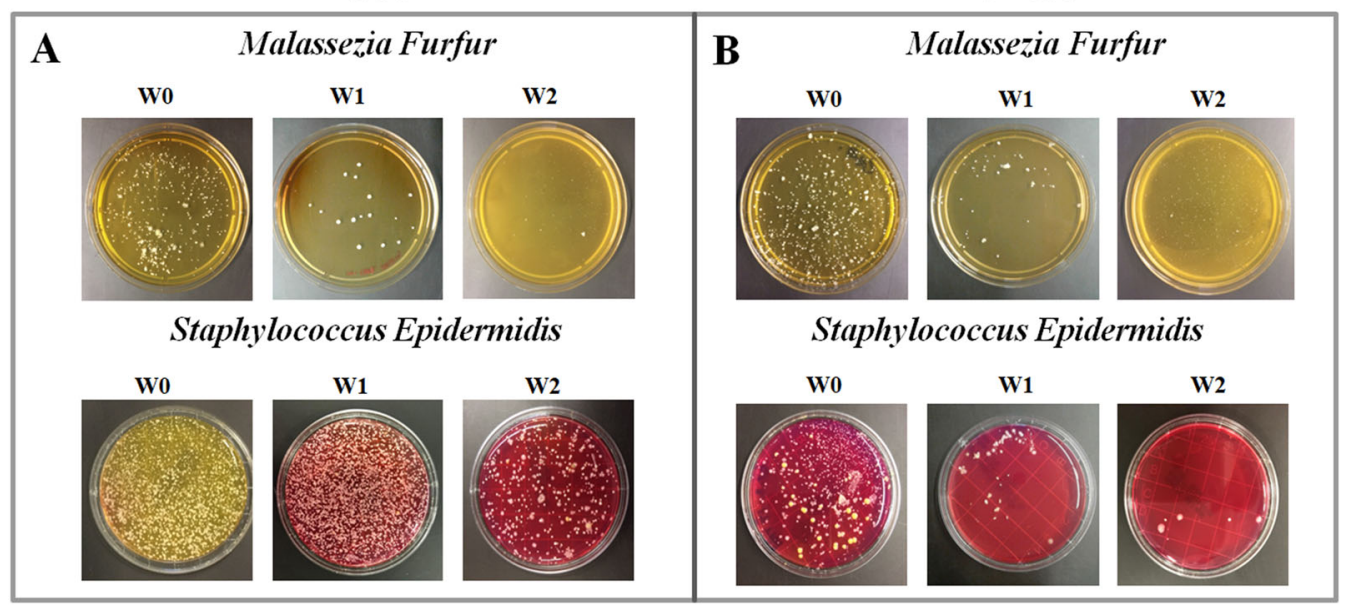

Fig. 3 Representative pictures of colony-forming units per dish at W0, W1 and W2 of Malassezia furfur and Staphylococcus epidermidis (SE) on face as well as on chest (a, b). The over growth of SE can cause the color change of the medium from red to yellow
A

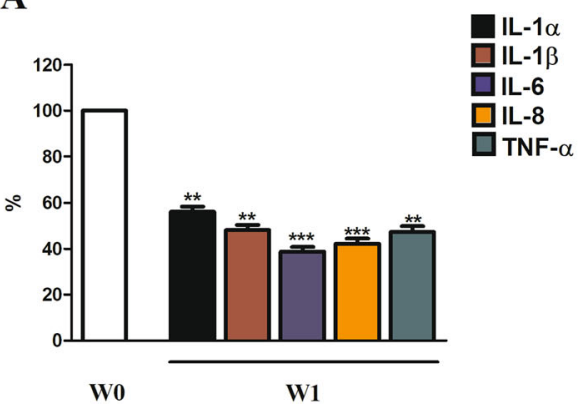

B

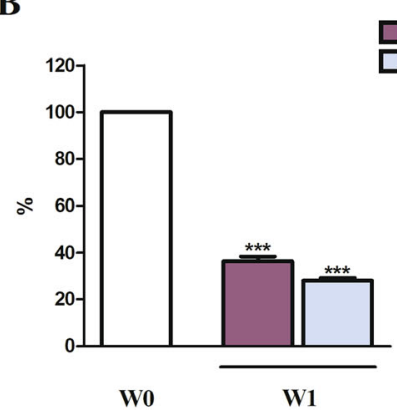

C HBD2 HBD3

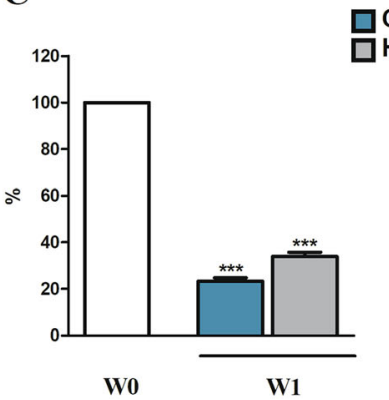

Fig. 4 Gene expression analysis in SD chest skin at baseline (W0) and after 1 week (W1) of treatment. Values are normalized to the housekeeping gene $18 \mathrm{~S}$ and expressed as mean \pm standard deviation. W0 represents

\section{DISCUSSION}

This study highlights the excellent clinical short-term efficacy of this NSC treatment in SD patients. Indeed, we report an improvement in signs and symptoms after just 1 week. All evaluated clinical parameters showed a progressive and significant decrease, thus obtaining great approval by the overall study population. Our findings are in line with a recent study that reported NSC clinical efficacy up to 8 weeks, with a significant reduction of erythema, pruritus and desquamation. In particular, they the expression of each evaluated gene and it is shown as $100 \%$. W 1 represents the percent of decrease with respect to W0. Statistical analyses were performed by Wilcoxon matched pairs test $\left({ }^{*} p<0.05 ;{ }^{* *} p<0.01,{ }^{* * *} p<0.001\right)$

observed a $90 \%$ reduction of VAS pruritus in the facial area, reporting $0.67 \pm 1.05 \mathrm{~cm}$ after 2 weeks of NSC, with respect to $6.9 \pm 1.68 \mathrm{~cm}$ at baseline [26]. Here, we observed an $85 \%$ reduction of VAS pruritus in the facial area, reporting $1.5 \pm 0.70 \mathrm{~cm}$ after 2 weeks of NSC treatment with respect to $9.6 \pm 0.14 \mathrm{~cm}$ at baseline. Moreover, we have provided the NSC effectiveness in term of VAS pruritus taking into account the chest area, too. Our data have shown a reduction of almost $70 \%$ with $6.7 \pm 0.35 \mathrm{~cm}$ at W0 and $2.2 \pm 0.35 \mathrm{~cm}$ at W2. Moreover, an important improvement efficacy for both face and chest in terms of IGA score was reported for 
all patients reaching a mild response at W1 (mean IGA score: 4.75 for face and 3.75 for chest) and excellent response at W2 (mean IGA score: 9.4 for face and 8.1 for chest). All these data are supported by Micali et al. In fact, they have previously evaluated IGA score after 8 weeks of NSC treatment, reporting good and excellent responses in $29.5 \%$ and $53 \%$ of patients, respectively [26]. Beyond the clinical efficacy, here we have also deepened the antifungal property in vivo, based on previous evidence in which this characteristic of NSC had already been tested in an ex vivo model [27]. Indeed, it was reported that at $24 \mathrm{~h}$ after application, NSC reduced the number of MF colonies by $92 \%$ compared to non-treated control skin. In line with these results [27], we have reported that NSC treatment on the face was able to decrease the number of MF colonies by $92.8 \%$ already after 1 week compared to W0, and a greater inhibition at W2, with the number of MF colonies reduced by $98 \%$, whereas the reduction registered for the chest area was 84\% and $99 \%$, at W1 and W2, respectively. Afterwards, we tested NSC effects on SE. Recent evidence suggested that a high colonization with SE, along with higher skin permeability barrier function, contributes to the occurrence of SD [28]. Here, we report that NSC treatment at W2 was able to decrease the number of SE colonies on the face by $75 \%$ compared to WO. An et al. [29] have demonstrated that fusidic acid treatment, applied for 2 weeks on the face, was able to decrease the number of SE colonies from 331 to 18 , achieving $95 \%$ compared to W0, whereas moisturizers increased the number of SE colonies compared to WO. It is worth mentioning that the decrease of SE colonies on the face (15.6\% W1, 75\% W2) was less pronounced than on the chest (92\% W1, 99\% W2). This difference might be due to higher colonization at baseline of SE on the face, since we have found approximately double the number of SE colonies on the face $(1600 \pm 60 \mathrm{cfu})$ with respect to the chest $(830 \pm 16 \mathrm{cfu})$. This preferential colonization is supported by Kloos et al. [30] who reported a predominant as well as persistent SE localization on different body areas, excluding the chest. Apart from investigating anti-fungal as well as anti-microbial effects of NSC treatment, we explored its impact on inflammation in SD. NSC was able to decrease gene expression of the main mediators of inflammation (IL- $1 \alpha$, IL-1 $\beta$, IL-6, IL-8, TNF- $\alpha$ ). This property could be related to lowering both fungal and microbial load. Our results fitted with the previously tested SD ex vivo model in which IL8 and TNF- $\alpha$ were decreased by NSC [27]. Pruritus was another feature addressed in this study. Here we showed that cathepsin S and CTS gene expression was strongly decreased by NSC treatment at $\mathrm{W} 1$, providing scientific rationale for the amelioration of pruritus sensation, which was confirmed by the patients' perception. Turlier et al. [31] have demonstrated that cathepsin $\mathrm{S}$ levels were decreased after 2 weeks of treatment with a shampoo in SD patients. The mechanism of action of this fragrance-free product may be related to multiple synergy of the main ingredients, that include: (1) piroctone olamine with anti-fungal and anti-microbial properties [32], (2) biosaccharide gum-2, an anti-inflammatory and soothing active ingredient protecting skin against external aggressions, that lead to dehydration [33] with recently demonstrated antifungal properties (patent pending WO2019020822), (3) stearyl glycyrrhetinate, a salt and ester of glycyrrhetinic acid that exhibits anti-inflammatory, soothing and anti-pruritic effects [34], and (4) zinc PCA which combines anti-inflammatory properties with anti-seborrheic efficacy [18]. We are aware that our study has some limitations: it was an open study from a single center, the number of patients was limited, and there was no control group treated with the vehicle without active ingredients. Moreover, only short-term use was evaluated. In conclusion, our assessment is that NSC is an effective and well tolerated treatment option for SD with anti-fungal, anti-microbial and anti-inflammatory properties.

\section{ACKNOWLEDGEMENTS}

We thank the patients for their participation in this study. 
Funding. ISDIN provided funding for the study and the Rapid Service Fee.

Authorship. All authors had full access to all of the data in this study and take complete responsibility for the integrity of the data and accuracy of the data analysis. All named authors meet the International Committee of Medical Journal Editors (ICMJE) criteria for authorship for this article, take responsibility for the integrity of the work as a whole, and have given their approval for this version to be published.

Authorship Contributions. Anna Balato, Giuseppina Caiazzo, Di Caprio, and Emanuele Scala performed the research, Anna Balato and Corinne Granger designed the research study. Anna Balato, Roberta Di Caprio, and Emanuele Scala analyzed the data. Anna Balato, Corinne Granger, Giuseppina Caiazzo, and Gabriella Fabbrocini wrote the paper.

Disclosures. Corinne Granger is an employee of ISDIN. Anna Balato has been a scientific consultant/speaker/clinical study investigator for AbbVie, Celgene, Janssen, EliLilly, ISDIN, Novartis, Pfizer, Sanofi and UCB. Gabriella Fabbrocini is a member of the journal's Editorial Board, and she has been a scientific consultant/speaker/clinical study investigator for AbbVie, Beiersdorf, Biodue, Boehringer Ingelheim, Coswell, Difa Cooper, Galderma, L'Oreal, Leo Pharma, Meda Pharma, Mertz Pharma, Pierre Fabre, and Sifarma. Giuseppina Caiazzo, Roberta Di Caprio, and Emanuele Scala have nothing to disclose.

Compliance with Ethics Guidelines. The experimental protocol was approved 14 March, 2018 by the Ethics Committee for Biomedical Activities "Carlo Romano" of University of Naples Federico II and conformed to the principles outlined in the Declaration of Helsinki of 1964, as revised in 2013, concerning human and animal rights. Each subject gave written informed consent before entering the study. The patient gave his permission for his photograph to be published in the manuscript.
Data Availability. The data sets generated and analyzed during the current study are available from the corresponding author on reasonable request.

Open Access. This article is distributed under the terms of the Creative Commons Attribution-NonCommercial 4.0 International License (http://creativecommons.org/licenses/ by-nc/4.0/), which permits any noncommercial use, distribution, and reproduction in any medium, provided you give appropriate credit to the original author(s) and the source, provide a link to the Creative Commons license, and indicate if changes were made.

\section{REFERENCES}

1. Borda L, Wikramanayake TC. Seborrheic dermatitis and dandruff: a comprehensive review. J Clin Invest Dermatol. 2015;2015:3-10.

2. Karakadze MA, Hirt PA, Wikramanayake TC. The genetic basis of seborrhoeic dermatitis: a review. J Eur Acad Dermatol Venereol. 2018;32:529-36.

3. Gupta AK, Nicol K, Batra R. Role of antifungal agents in the treatment of seborrheic dermatitis. Am J Clin Dermatol. 2004;5:417-22.

4. Schwartz JR, Messenger AG, Tosti A, Todd G, Hordinsky M, Hay RJ, et al. A comprehensive pathophysiology of dandruff and seborrheic dermatitistowards a more precise definition of scalp health. Acta Derm Venereol. 2013;93:131-7.

5. Hay RJ. Malassezia dandruff and seborrhoeic dermatitis: an overview. Br J Dermatol. 2011;165:2-8.

6. Ro BI, Dawson TL. The role of sebaceous gland activity and scalp microfloral metabolism in the etiology of seborrheic dermatitis and dandruff. J Investig Dermatol Symp Proc. 2005;10:194-7.

7. Heng MC, Henderson CL, Barker DC, Haberfelde G. Correlation of Pityrosporum ovale density with clinical severity of seborrheic dermatitis as assessed by a simplified technique. J Am Acad Dermatol. 1990;23:82-6.

8. McGinley KJ, Leyden JJ, Marples RR, Kligman AM. Quantitative microbiology of the scalp in nondandruff, dandruff, and seborrheic dermatitis. J Invest Dermatol. 1975;64:401-5. 
9. DeAngelis YM, Saunders CW, Johnstone KR, Reeder NL, Coleman CG, Kaczvinsky JR, et al. Isolation and expression of a Malassezia globosa lipase gene, LIP1. J Invest Dermatol. 2007;127:2138-46.

10. Plotkin LI, Squiquera L, Mathov I, Galimberti R, Leoni J. Characterization of the lipase activity of Malassezia furfur. J Med Vet Mycol. 1996;34:43-8.

11. Warner RR, Schwartz JR, Boissy Y, Dawson TL Jr. Dandruff has an altered stratum corneum ultrastructure that is improved with zinc pyrithione shampoo. J Am Acad Dermatol. 2001;45:897-903.

12. Faergemann J, Bergbrant IM, Dohse M, Scott A, Westgate G. Seborrhoeic dermatitis and Pityrosporum (Malassezia) folliculitis: characterization of inflammatory cells and mediators in the skin by immunohistochemistry. $\mathrm{Br} \mathrm{J}$ Dermatol. 2001;144:549-56.

13. Tanaka A, Cho O, Saito C, Saito M, Tsuboi R, Sugita T. Comprehensive pyrosequencing analysis of the bacterial microbiota of the skin of patients with seborrheic dermatitis. Microbiol Immunol. 2016;60:521-6.

14. Piquero-Casals J, Hexsel D, Mir-Bonafé JF, RozasMuñoz E. Topical non-pharmacological treatment for facial seborrheic dermatitis. Dermatol Ther (Heidelb). 2019;9:469-77.

15. Dall'Oglio F, Tedeschi A, Fusto CM, Lacarrubba F, Dinotta F, Micali G. A novel cosmetic antifungal/ anti-inflammatory topical gel for the treatment of mild to moderate seborrheic dermatitis of the face: an open-label trial utilizing clinical evaluation and erythema-directed digital photography. G Ital Dermatol Venereol. 2017;152:436-40.

16. Schmidt-Rose T, Braren S, Fölster H, Hillemann T, Oltrogge B, Philipp P, et al. Efficacy of a piroctone olamine/climbazol shampoo in comparison with a zinc pyrithione shampoo in subjects with moderate to severe dandruff. Int $\mathrm{J}$ Cosmet Sci. 2011;33(3):276-82.

17. Granger C, Goni F, Martinez G, Garre A. Ex-vivo determination of antifungal activity of a new prescription non-steroidal facial cream against Malassezia furfur in human skin explants. Skin J Cutan Med. 2017;1:137.

18. Takino Y, Okura F, Kitazawa M, Iwasaki K, Tagami H. Zinc L-pyrrolidone carboxylate inhibits the UVA induced production of matrix metalloproteinase-1 by in vitro cultured skin fibroblasts, whereas it enhances their collagen synthesis. Int J Cosmet Sci. 2012;34(1):23-8.

19. Rostan EF, DeBuys HV, Madey DL, Pinnell SR. Evidence supporting zinc as an important antioxidant for skin. Int J Dermatol. 2002;41:606-11.
20. Lang C, Murgia C, Leong M, Tan LW, Perozzi G, Knight D, et al. Antiinflammatory effects of zinc and alterations in zinc transporter mRNA in mouse models of allergic inflammation. Am J Physiol Lung Cell Mol Physiol. 2007;292:L577-84.

21. Valdera-Martinez P, Garre A, Granger C. Efficacy and tolerance of a new non-steroidal prescription cream in the treatment of mild facial seborrheic dermatitis. Skin J Cutan Med. 2017;1:s134.

22. Oyama K, Kawada-Matsuo M, Oogai Y, Hayashi T, Nakamura N, Komatsuzawa H. Antibacterial effects of glycyrrhetinic acid and its derivatives on Staphylococcus aureus. PLoS One. 2016;11(11):e0165831.

23. Koca R, Altinyazar HC, Eştürk E. Is topical metronidazole effective in seborrheic dermatitis? A double-blind study. Int J Dermatol. 2003;42:632-5.

24. Lembo S, Di Caprio R, Balato A, Caiazzo G, Fabbrocini G, Skroza N, et al. The increase of mTOR expression is consistent with FoxO1 decrease at gene level in acne but not in psoriasis. Arch Dermatol Res. 2019. https://doi.org/10.1007/s00403019-01959-0. [Epub ahead of print].

25. Livak KJ, Schmittgen TD. Analysis of relative gene expression data using real-time quantitative PCR and the 2(-Delta Delta $\mathrm{C}(\mathrm{T})$ ) method. Methods. 2001;25:402-8.

26. Dall' Oglio F, Lacarrubba F, Luca M, Boscaglia S, Granger C, Micali G. Clinical and instrumental evaluation of a new topical non-corticosteroid antifungal/anti-inflammatory/antiseborrheic combination cream for the treatment of mild-to-moderate facial seborrheic dermatitis. Clin Cosmet Investig Dermatol. 2019;12:103-8.

27. Granger C, Balato A, Goñi-de-Cerio F, Garre A, Narda M. Novel non-steroidal facial cream demonstrates antifungal and anti-inflammatory properties in ex vivo model for seborrheic dermatitis. Dermatol Ther (Heidelb). 2019;9:571-8.

28. Dessinioti C, Katsambas A. Seborrheic dermatitis: etiology, risk factors, and treatments: facts and controversies. Clin Dermatol. 2013;31:343-51.

29. An Q, Sun M, Qi RQ, Zhang L, Zhai JL, Hong YX, et al. High Staphylococcus epidermidis colonization and impaired permeability barrier in facial seborrheic dermatitis. Chin Med J (Engl). 2017;130:1662-9.

30. Kloos WE, Musselwhite MS. Distribution and persistence of Staphylococcus and Micrococcus species and other aerobic bacteria on human skin. Appl Microbiol. 1975;30:381-5. 
31. Turlier V, Viode C, Durbise E, Bacquey A, LeJeune $\mathrm{O}$, Oliveira Soares R, et al. Clinical and biochemical assessment of maintenance treatment in chronic recurrent seborrheic dermatitis: randomized controlled study. Dermatol Ther (Heidelb). 2014;4:43-59.

32. Okokon E, Verbeek J, Ruotsalainen J, Ojo O, Bakhoya V. Topical antifungals for seborrhoeic dermatitis. Cochrane Database Syst Rev. 2015;4:CD008138.
33. Marseglia A, Licari A, Agostinis F, Barcella A, Bonamonte D, Puviani $M$, et al. Local rhamnosoft, ceramides and L-isoleucine in atopic eczema: a randomized, placebo controlled trial. Pediatr Allergy Immunol. 2014;25:271-5.

34. Asl MN, Hosseinzadeh H. Review of pharmacological effects of Glycyrrhiza sp. and its bioactive compounds. Phytother Res. 2008;22:709-24. 